\title{
La nouvelle vague de COVID-19 sera-t-elle un tsunami pour la chirurgie?
}

Les opinions exprimées dans cet éditorial sont celles de l'auteur et ne représentent pas nécessairement celles de l'Association médicale canadienne ou ses filiales.

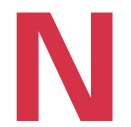

ous pensions nous en sortir plutôt bien, avec cette histoire de pandémie. Le Canada était en avance sur une bonne partie du monde en matière de vaccination. Nous nous étions habitués à la distanciation physique et aux masques; ils pouvaient bien rester. Même l'imposition de passeports vaccinaux pour avoir accès à plus de lieux intérieurs semblait acceptée par la majorité. Mais peut-être avons-nous été trop optimistes, trop tôt, car voilà arrivée la quatrième vague de la COVID-19 que nous craignions.

Comme l'a annoncé l'administratrice en chef de la santé publique du Canada, les données de surveillance nationales montrent qu'une quatrième vague frappe actuellement le pays et que les cas sont à la hausse dans certaines régions : la moyenne de cas quotidiens sur 7 jours au 29 septembre était de 4358 . Nous sommes donc revenus au niveau de mai $2021^{1}$, bien loin de la baisse attendue. Le variant à l'origine de cette remontée des cas est le variant Delta, apparemment plus contagieux que les précédents, et c'est chez les non-vaccinés que l'on retrouve les patients les plus gravement malades.

Quoi qu'en disent les groupes anti-vaccination, les ressources de soins de santé sont drainées par cette résurgence. Les lits de soins intensifs et d'hôpital se trouvent maintenant occupés par des personnes non vaccinées atteintes de la COVID-19. Les ressources sont également mises à mal par l'épuisement professionnel qui sévit chez les fournisseurs de soins de santé, y compris chez le personnel auxiliaire. Le problème de l'épuisement professionnel est exacerbé par l'absence du personnel des ressources humaines, qui a été renvoyé à la maison en raison de la pandémie. Le roulement normal requiert un processus d'embauche constant, processus qui a été mis en pause avec tout le reste.

Ignorer la situation ne fonctionne pas. La réouverture de l'Alberta sans distanciation physique ni passeport vaccinal en est un exemple sans équivoque, et la pénurie extrême que connaît le système de santé de la province sert d'avertissement aux autres. L'Ontario a pour sa part abandonné sa réouverture rapide au profit de mesures resserrées, dans l'espoir de tuer la quatrième vague dans l'œuf.

Les opérations chirurgicales non urgentes sont en attente ou fortement limitées dans la plupart des pro- vinces. Les opérations des extrémités, les réparations herniaires et les chirurgies bariatriques ont été reportées. Les listes de patients attendant ces opérations, déjà trop longues, ne feront que gonfler davantage. On se limite encore aux chirurgies oncologiques prioritaires, et les opérations sur les personnes dont la vie ou un membre est en danger se font toujours, mais à un rythme légèrement plus lent. Et que fait-on des patients atteints de fractures ou d'insuffisance vasculaire grave? Qu'ils soient confinés à leur civière ou non, ces patients s'exposent à une mortalité et à une morbidité bien démontrées lorsque la chirurgie tarde trop. Pourtant, on rationne les soins, directement ou indirectement, par manque de lits.

Les gouvernements sont en train de prendre conscience des risques. En 2021, le Bureau de la responsabilité financière de l'Ontario estime qu'il en coûtera 1,3 milliard de dollars pour gérer l'arriéré des chirurgies découlant de la pandémie ${ }^{2}$. Il admet dans son rapport que le financement prévu est insuffisant, l'Ontario ayant alloué 610 millions de dollars à cette tâche ${ }^{2}$. Il faudra également d'autres fonds pour les examens critiques et certaines chirurgies non urgentes prioritaires, mais dans la situation actuelle, les patients sont victimes d'une négligence presque totale. Nous avons besoin d'une entente nationale, pilotée par des chirurgiens, sur les mesures à prendre pour que les soins aux patients ne s'effondrent pas complètement. Les gouvernements provinciaux devront la signer et exécuter le plan, ce qui prendra probablement plus de 5 ans - plus que la plupart des mandats politiques. Nous devons attaquer aux problèmes actuels et aux conditions qui y ont mené pour changer véritablement les soins prodigués à nos patients. Et il faut agir vite, sinon l'avenir s'annonce bien sombre.

\section{Edward J. Harvey, MD, MSc \\ Corédacteur en chef, fournal canadien de chirurgie}

Intérêts concurrents: E.J. Harvey est médecin chef chez Greybox Solutions; il est cofondateur et directeur de l'innovation médicale de NXTSens Inc, cofondateur et médecin chef de MY01 Inc. et cofondateur et directeur de Strathera Inc. Il bénéficie du soutien institutionnel de J \& J, DePuy Synthes, Stryker et Zimmer, et il fait partie du conseil d'administration de l'Orthopedic Trauma Association et de l'Association canadienne d'orthopédie. 
Propriété intellectuelle du contenu : Il s'agit d'un article en libre accès distribué conformément aux modalités de la licence Creative Commons Attribution (CC BY-NC-ND 4.0), qui permet l'utilisation, la diffusion et la reproduction dans tout médium à la condition que la publication originale soit adéquatement citée, que l'utilisation se fasse à des fins non commerciales (c.-à-d., recherche ou éducation) et qu'aucune modifcation ni adaptation n'y soit apportée. Voir : https://creativecommons.org/licenses/by-nc -nd/4.0/deed.fr.

Citation: Can J Surg 2021 October 26; 64(5). doi: 10.1503/ cjs. 017721

\section{Références}

1. COVID cases in Canada tracker: how many new cases of COVID-19 today? Global News 2020 Mar. 9 [updated 2021 Oct. 6]. Accessible ici : https://globalnews.ca/news/6649164/canada -coronavirus-cases/ (consulté le 6 oct. 2021).

2. Financial Accountability Office of Ontario. Ministry of Health 2021 spending plan review. FAO; 2021. Accessible ici : https:// www.fao-on.org/web/default/files/publications/FA2 101-1\%20 Health \% 20 Estimates/Health \% 20 Spending \% 20 Plan \% 20 Review\% 20 Presentation-EN.pdf (consulté le $1^{\text {er }}$ oct. 2021). 